\title{
SMYD3 promotes colon adenocarcinoma (COAD) progression by mediating cell proliferation and apoptosis
}

\author{
FU-REN YUE*, ZHI-BIN WEI*, RUI-ZHEN YAN*, QIU-HONG GUO, BING LIU, JING-HUI ZHANG and ZHENG LI \\ Department of Clinical Laboratory, Tianjin Baodi Hospital, Tianjin 301800, P.R. China
}

Received April 25, 2020; Accepted July 24, 2020

DOI: $10.3892 /$ etm.2020.9139

\begin{abstract}
Colon adenocarcinoma (COAD) is a type of common malignant tumor originating in the digestive tract. Recently, targeted therapy has had significant effects on the treatment of COAD. However, more effective molecular targets need to be developed. SET and MYND domain-containing protein 3 (SMYD3) is a type of methyltransferase which methylates histone and non-histone proteins. The effects of SMYD3 on cancer progression and metastasis have been widely revealed. However, its possible role in COAD remains unclear. The current study demonstrated that SMYD3 expression was upregulated in human COAD tissues via analyzing the The Cancer Genome Atlas (TCGA) database and the immunohistochemical assays. Furthermore, the expression of SMYD3 was correlated with prognosis and tumor stage $(\mathrm{P}=0.038)$ in patients with COAD. Colony formation, MTT, FCM assays and animal assays indicated SMYD3 affected the proliferation, apoptosis and the cell cycle of COAD cells in vitro and promoted tumor growth in mice in vivo. In summary, the results demonstrated the effects of SMYD3 on COAD progression and we hypothesized that SMYD3 is a novel molecular target for COAD treatment.
\end{abstract}

\section{Introduction}

Colon adenocarcinoma (COAD) is a common malignant tumor in the digestive tract (1). The etiology of COAD remains unclear. However, COAD is often associated with a fatty diet (2). The lack of significant symptoms in the early stages of COAD increases the difficulty of diagnosis. The existing treatment methods for advanced stages of COAD, including

Correspondence to: Dr Jing-Hui Zhang or Dr Zheng Li, Department of Clinical Laboratory, Tianjin Baodi Hospital, 8 Guangchuan Road, Tianjin 301800, P.R. China

E-mail: bdyyzhjh@163.com

E-mail: tianshangxizang18@163.com

${ }^{*}$ Contributed equally

Key words: SET and MYND domain-containing protein, SMYD3, colon adenocarcinoma, proliferation, apoptosis, molecular target radiotherapy, chemotherapy and surgical resection, have little effect on this disease, leading to a high incidence of mortality, with $>600,000$ patients succumbing to COAD worldwide every year $(3,4)$. Recently, targeted therapy has had a significant effect on COAD (4). However, it is crucial to discover more effective therapeutic targets to improve patient survival.

Histone methyltransferases are a group of epigenetic regulators which promote histone lysine methylation (5). SET and MYND domain-containing protein 3 (SMYD3) is a type of methyltransferase that methylates histones 3 or 4 at lysine 4 or 20 , respectively (H3K4 or H4K20) $(6,7)$. A previous study indicated that SMYD3 promoted homologous recombination by altering H3K4-mediated gene expression (8). Chitooligosaccharides regulate glucose-Lipid metabolism by inhibiting SMYD3 and mediating gut microflora (9). Additionally, SMYD3 is recruited by Ebola virus nucleoprotein to facilitate viral mRNA transcription (10).

The effects of SMYD3 on cancer progression and metastasis have been widely investigated (11-14). SMYD3 was found to be overexpressed in several types of tumors, including bladder cancer, gastric cancer, lung cancer and pancreatic cancer (15-20). In breast cancer, SMYD3 contributes to epithelial-mesenchymal transition progression (12). Additionally, SMYD3 promoted cancer progression and intrahepatic metastasis of hepatocellular carcinoma by regulating CDK2 and MMP2 expression (16,21). Although the involvement of SMYD3 in cancer progression has been widely reported, its possible role in COAD remains unclear.

The present study demonstrated the high expression of SMYD3 in human COAD tissues and revealed that high expression of SMYD3 was correlated with the prognosis and clinical pathological features of patients with COAD. Furthermore, the results demonstrated the effects of SMYD3 on the proliferation of COAD in vitro and in mice. Therefore, we hypothesized that SMYD3 serves as a promising molecular target for COAD treatment.

\section{Materials and methods}

TCGA database analysis. The TCGA database analysis was performed through GEPIA (http://gepia.cancer-pku.cn/detail. php?gene=SMYD3/) to analyze the data with a threshold of $\mathrm{P}<0.05$ and $\log \mathrm{FC}>1$ or $<-1$ for the differential genes. Additionally, the median value was used as the basis for dividing the patients into two groups for the Kaplan-Meier (KM) 
survival analysis, and the dotted line indicated the $95 \%$ confidence interval.

Antibodies, primers and short hairpin (sh)RNA plasmids. Anti-SMYD3 (for IHC assays, 1:200; for immunoblotting assays, 1:1,000; cat. no. ab187149) and anti- $\beta$-actin (1:3,000; cat. no. ab8226) were purchased from Abcam.

The quantitative PCR (qPCR) primer sequences used were as follows: SMYD3 forward, 5'-CGCGTCGCCAAATACTGT AGT-3' and reverse, 5'-CAAGAAGTCGAACGGAGTCTG-3'; and GAPDH forward, 5'-CGACCACTTTGTCAAGCTCA-3' and reverse, 5'-GGTTGAGCACAGGGTACTTTATT-3'. The shRNA plasmids of SMYD3 were purchased from Addgene.

Human tissue samples and analysis. A total of 92 human COAD tissues and adjacent normal tissues $(3 \mathrm{~cm}$ from tumor tissues) were collected from patients at Tianjin Baodi Hospital, Tianjin, China. The clinicopathological characteristics are listed in Table I.

To explore the correlation between SMYD3 expression and the progression of COAD, immunohistochemical (IHC) assays were performed. Briefly, resin was used for tissue embedding tissue, and $3 \mu \mathrm{m}$ sections were performed. Sections were subsequently fixed with $4 \%$ paraformaldehyde (PFA) at room temperature for $20 \mathrm{~min}$ and blocked with $2 \%$ BSA for $20 \mathrm{~min}$ at room temperature. Slides were incubated with SMYD3 antibodies (1:200; cat. no. ab187149; Abcam) at room temperature for $2 \mathrm{~h}$. Sections were then incubated with mice or rabbit biotinylated secondary antibodies (1:500; cat. no. ab201485; cat. no. ab99807, respectively; Abcam) at room temperature for $1.5 \mathrm{~h}$. Diaminobenzidine was used as the chromogen substrate.

SMYD3 was mainly located in the cytoplasm of COAD tissues. The proportion of positive stained cells was graded as follows: $0,0 \%$ stained cells; $1,1-30 \%$ stained cells; 2, 31-70\% stained cells; 3, 71-100\% stained cells. Staining intensity was evaluated on a score of 0 (no or low staining), 1 (modest staining) and 2 (high staining). Tissues were divided into high or low SMYD3 expression groups according to the staining index: Score of staining intensity + score of staining cells $\%$. A staining index of $<3$ indicated low SMYD3 expression and $\geq 3$ high expression.

Cell culture and transfection. Human colorectal cancer cell lines, HCT116 and HT-29, were purchased from the American Type Culture Collection. HCT116 and HT-29 cells were incubated in RPMI-1640 medium (Gibco; Thermo Fisher Scientific, Inc.) supplemented with 10\% FBS (Gibco; Thermo Fisher Scientific, Inc.) in a 5\% $\mathrm{CO}_{2}$ incubator. SMYD3 shRNA plasmids were transfected into HCT116 and HT-29 cells using Lipofectamine 2000 (cat. no. 11668019; Invitrogen; Thermo Fisher Scientific, Inc.). The shRNA target sequences were presented as follows: Control: 5'-CCUACAUCCCGAUCGAUG AUGUUGA-3'; SMYD3: 5'-AGCCTGATTGAAGATTTG ATT-3'.

The further in vitro assays were performed $24 \mathrm{~h}$ after the transfection. Stably SMYD3 depleted HCT116 cells were screened by the infection of its shRNA lentivirus and used for in vivo tumor growth assays.

qPCR assays. TRIzol (cat. no. 15596026; Invitrogen; Thermo Fisher Scientific, Inc.) was used to extract total RNA from
HCT116 and HT-29 cells. RNA was reverse transcribed using M-MLV reverse transcriptase (cat. no. M1701; Promega Corporation). Total mRNA was reverse transcribed to produce cDNA using a cDNA synthesis system (cat. no. 6110A; Takara Bio, Inc.). qPCR was performed using a SYBR Ex Taq kit (cat. no. 638319; Takara Bio, Inc.), which was used according to the manufacturer's protocol. The reaction conditions were as follows: Pre-denaturation, $95^{\circ} \mathrm{C}, 3 \mathrm{~min}$; Denaturation, $95^{\circ} \mathrm{C}$, $30 \mathrm{sec}$; Annealing, $58^{\circ} \mathrm{C}, 30 \mathrm{sec}$; Extension, $72^{\circ} \mathrm{C}, 30 \mathrm{sec}$. There are 35 cycles. The $2^{-\Delta \Delta C q}$ method was used to quantify results (22). SMYD3 expression levels were normalized to GAPDH.

Immunoblotting assays. HCT116 and HT-29 cells or COAD tissues were lysed using RIPA buffer (cat. no. 9800; Cell Signaling Technology, Inc.). BSA method was used for protein determination, and $20 \mu 1$ sample was loaded in each lane at a concentration of $1 \mathrm{mg} / \mathrm{ml}$. Total protein was separated using 10\% SDS-PAGE and transferred onto nitrocellulose membranes. Membranes were blocked with 5\% milk in TBST at room temperature for $2 \mathrm{~h}$ and incubated with primary antibodies for the detection of SMYD3 (1:1,000; cat. no. ab187149; Abcam) and $\beta$-actin (1:3,000; cat. no. Ab8226; Abcam) at room temperature for $2 \mathrm{~h}$ at room temperature. Following this, membranes were incubated with horseradish peroxidase-conjugated secondary antibodies for $45 \mathrm{~min}$ at room temperature. Signals were detected using an ECL kit $\left(\right.$ Novex $^{\mathrm{TM}}$ ECL Chemiluminescent Substrate Reagent kit; Thermo Fisher Scientific, Inc.) according to the manufacturer's protocol.

Colony formation assays. HCT116 and HT-29 cells were added into six-well culture plates with the seeding density of $1,000 \mathrm{cell} /$ well and transfected with control or SMYD3 shRNA plasmids. The medium was replaced with fresh medium every 3 days. After 2 weeks, cells were fixed with PFA for $30 \mathrm{~min}$ at room temperature, stained with $0.2 \%$ crystal violet at room temperature for $20 \mathrm{~min}$ and washed twice with PBS. Colony numbers were counted manually.

MTT assays. HCT116 and HT-29 cells were plated into 96-well plates (seeding density, $2 \times 10^{3} /$ well), respectively, transfected with control or SMYD3 shRNA plasmids using Lipofectamine 2000 (cat. no. 11668019; Invitrogen; Thermo Fisher Scientific, Inc.) and incubated at $37^{\circ} \mathrm{C}$ for 2 days. Following this, cells were incubated with MTT for $4 \mathrm{~h}$ at room temperature and removed the culture medium. After washing twice with PBS buffer, $150 \mu$ DMSO was added into each well to extract cells. OD values were measured with a microplate reader at a wavelength of $570 \mathrm{~nm}$ and the relative proliferation degrees were assessed.

Flow cytometry (FCM) assays. For the detection of cell apoptosis, HCT116 or HT-29 cells were fixed with $70 \%$ ethyl alcohol for $\mathrm{h}$ hours at $-20^{\circ} \mathrm{C}$ and treated with Annexin V-FITC/propidium iodide (PI) using a kit (Annexin V-FITC Apoptosis Detection Kit, APOAF; Sigma-Aldrich; Merck KGaA) for $20 \mathrm{~min}$ at room temperature. Cells were detected using a FACS Calibur flow cytometer (FACSAria III; BD Biosciences), and the analysis software was the supporting software of the flow cytometer. The apoptosis cells, including early and late apoptotic cells were analyzed. 
Table I. Correlation between SMYD3 expression and the clinical pathological features of 92 patients with colon adenocarcinoma.

\begin{tabular}{|c|c|c|c|c|c|}
\hline \multirow[b]{2}{*}{ Feature } & \multirow[b]{2}{*}{ No. } & \multicolumn{2}{|c|}{ SMYD3 expression } & \multirow[b]{2}{*}{$\chi^{2}$} & \multirow[b]{2}{*}{ P-value } \\
\hline & & $\begin{array}{l}\text { Low } \\
n=25\end{array}$ & $\begin{array}{l}\text { High } \\
n=67\end{array}$ & & \\
\hline Age (years) & & & & 1.689 & 0.194 \\
\hline$<55$ & 47 & 10 & 37 & & \\
\hline$\geq 55$ & 45 & 15 & 30 & & \\
\hline Sex & & & & 0.038 & 0.846 \\
\hline Male & 50 & 14 & 36 & & \\
\hline Female & 42 & 11 & 31 & & \\
\hline Tumor stage & & & & 4.318 & $0.038^{\mathrm{a}}$ \\
\hline $\mathrm{T} 2$ & 29 & 12 & 17 & & \\
\hline $\mathrm{T} 3 / \mathrm{T} 4$ & 63 & 13 & 50 & & \\
\hline Tumor grade & & & & 1.234 & 0.267 \\
\hline Low & 22 & 8 & 14 & & \\
\hline High & 70 & 17 & 53 & & \\
\hline \multicolumn{6}{|l|}{ Lymph node } \\
\hline metastasis & & & & 2.425 & 0.119 \\
\hline Yes & 49 & 10 & 39 & & \\
\hline No & 43 & 15 & 28 & & \\
\hline Metastasis & & & & 0.398 & 0.528 \\
\hline Yes & 38 & 9 & 29 & & \\
\hline No & 54 & 16 & 38 & & \\
\hline
\end{tabular}

SMYD3, SET and MYND domain-containing protein 3; T, tumor. ${ }^{\mathrm{P}} \mathrm{P}<0.05$.

For cell cycle assays, cells were fixed with $70 \%$ ethyl alcohol for $24 \mathrm{~h}$ at $-20^{\circ} \mathrm{C}$ and incubated with $50 \mu \mathrm{g} / \mathrm{ml} \mathrm{PI}$ at $37^{\circ} \mathrm{C}$ for $30 \mathrm{~min}$. Samples were detected using a flow cytometer (FACSAria III; BD Biosciences). The percentage of cells at different phases (Including the G1, S and G2/M phases) were analyzed.

Tumor growth assays. All animal experiments were approved by the Institutional Animal Care and Use Committee of Tianjin Baodi Hospital, Tianjin, China. Female BALB/c nude mice (10-week-old) were supplied by Beijing Weitong Lihua Experimental Animal Technology Co., Ltd. Mice weight was approximately 22-24 g. Mice were fed with food and water ad libitum, and were fed at Specific Pathogen Free (SPF) level at $20^{\circ} \mathrm{C}$ and a humidity of $60 \%$, alternating between light and dark for 12 h. In vivo assays were performed to according to the following criteria. A total of 16 athymic nude mice were included in control $(n=8)$ and shRNA treatment $(n=8)$ groups. None of the mice died during the study.

HCT116 cells were stably infected with control or SMYD3 shRNA lentiviruses, as previously described. Subsequently, $\sim 2 \times 10^{6}$ HCT116 cells infected with the indicated lentiviruses were subcutaneously implanted into the mice. Tumors formation began at 14 days post-implantation. All mice were maintained until the assays were finished. Tumor volumes were measured every 3 days from the third week through a vernier caliper after 14 days.
After 29 days, all mice were sacrificed via cervical dislocation and tumor tissues were subsequently excised A lack of a heartbeat was established to confirm death. Adequate humanitarian care was given.

Tumors were isolated and photographed, and tumor growth curves were calculated and compared. Tumor volume was calculated as follows: Tumor volume $\left(\mathrm{mm}^{3}\right)=$ tumor length $(\mathrm{mm}) \mathrm{x}$ tumor width $(\mathrm{mm})^{2} / 2$. In terms of weight, mice gained no more than $5 \%$ and lost no more than $8 \%$ of their body weight. The weight of different groups of mice were not significantly different between groups during the whole feeding period. The maximum diameter of tumors was $\sim 12 \mathrm{~mm}$ and maximum tumor weight was $\sim 4 \%$ of total body weight.

Statistical analysis, GraphPad software (version 5.0; GraphPad Software, Inc.) was used for statistical analysis. Data are presented as mean \pm SD. The correlation between clinical pathological features and SMYD3 expression was analyzed by Pearson's $\chi^{2}$ analysis. Student's t-test was used for statistical comparisons. $\mathrm{P}<0.05$ was considered to indicate a statistically significant difference.

\section{Results}

SMYD3 expression is enhanced in human COAD tissues and correlates with the prognosis and clinical features of patients with COAD. To assess the potential effects of SMYD3 in the 
A

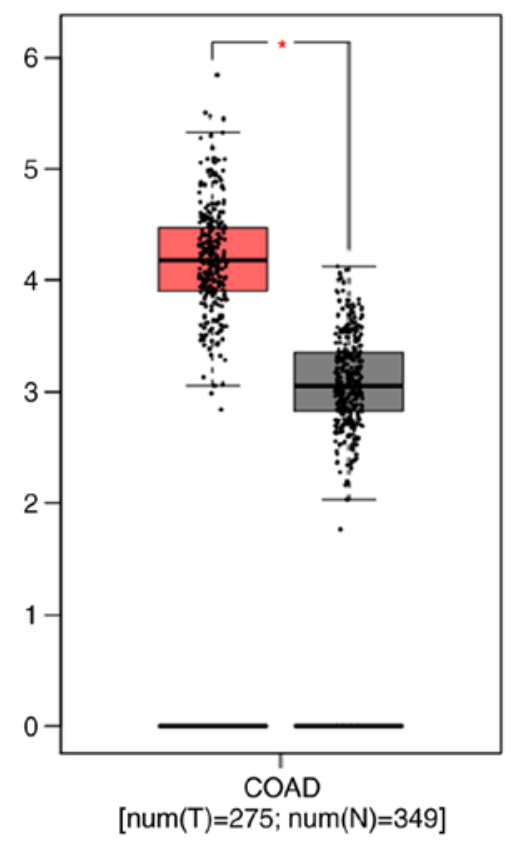

B
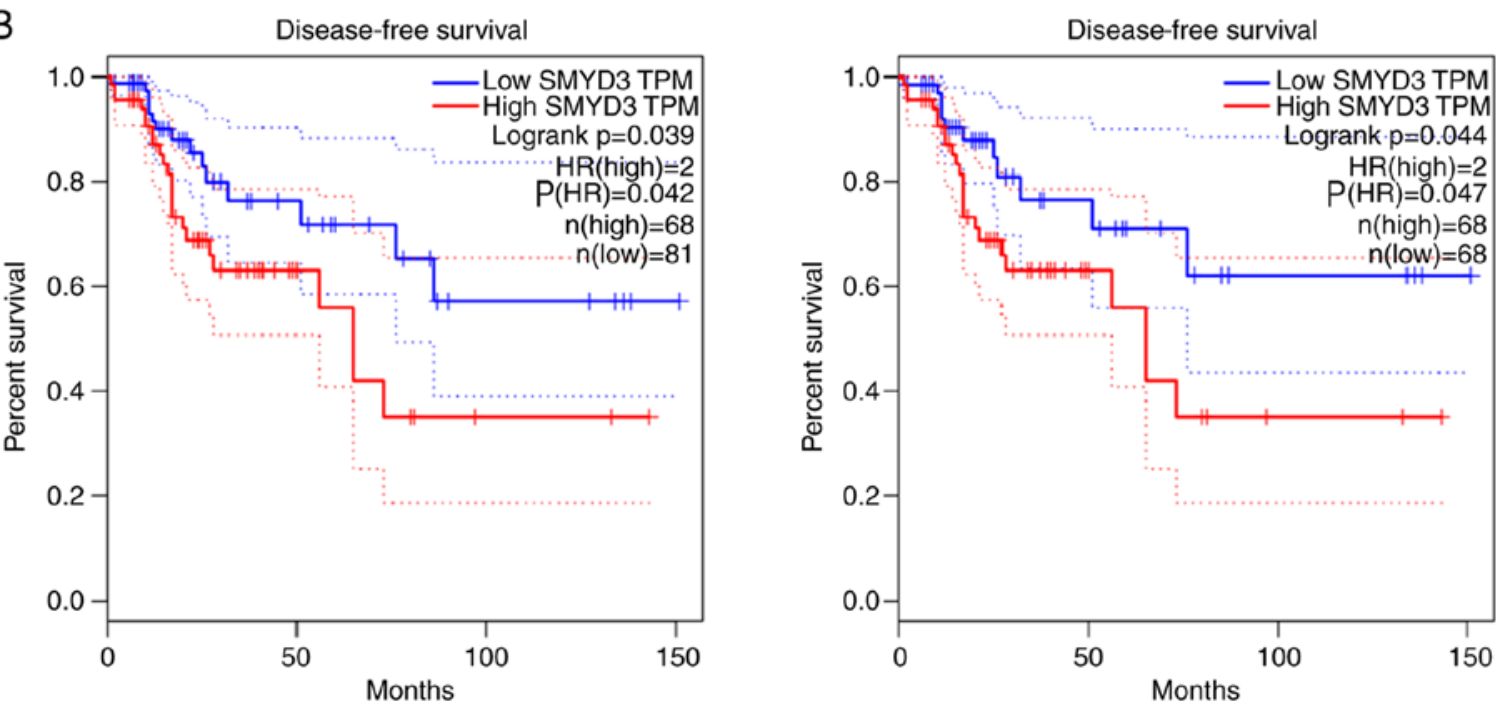

Figure 1. SMYD3 mRNA levels are increased in COAD tissues and correlate with the prognosis of patients with COAD. (A) SMYD3 mRNA levels in COAD tissues and normal tissues were assessed using TCGA database. (B) SMYD3 mRNA levels were correlated with the disease free survival rates of two sets of patients with COAD, according to the TCGA database. SMYD3, SET and MYND domain-containing protein 3; COAD, colon adenocarcinoma; TCGA, The Cancer Genome Atlas; T, tumor; N, normal. ${ }^{*} \mathrm{P}<0.05$. TRM, transcripts perkilobase million.

progression of COAD, the mRNA levels of SMYD3 in tumor tissues and normal tissues were assessed using The Cancer Genome Atlas (TCGA) database. A total of 275 COAD tissues and 349 adjacent tissues were included. The results demonstrated significantly high SMYD3 mRNA levels in human COAD tissues compared with adjacent normal tissues (Fig. 1A). Furthermore, the correlation between SMYD3 expression and prognosis of patients with COAD was evaluated using the TCGA database. K-M survival analysis was performed to assess whether SMYD3 expression impaired the disease-free survival rates of patients with COAD based on the TCGA database. Also according to the TCGA database, two sets of patients were included $(n=149$ and $\mathrm{n}=136$ ). Notably, SMYD3 expression was correlated with the disease-free survival rates of patients with COAD $(\mathrm{P}=0.042$ and $\mathrm{P}=0.047$, respectively; Fig. 1B). Therefore, the results indicated high SMYD3 expression in human COAD tissues and that this expression was correlated with the prognosis of COAD.

Subsequently, the expression of SMYD3 in COAD tissues and adjacent normal tissues collected at the hospital was evaluated. The results of IHC assays revealed that SMYD3 was mainly located in the cytoplasm of tumor tissues (Fig. 2). Furthermore, high expression of SMYD3 in COAD tissues compared to the adjacent tissues was observed, which was consistent with the data presented in Fig. 1.

The correlation analysis between SMYD3 expression and clinical features of patients with COAD. Following this, the clinical pathological features of 92 patients with COAD were analyzed (Table I). Patients were divided into two groups according to a scoring method: Low or high SMYD3 

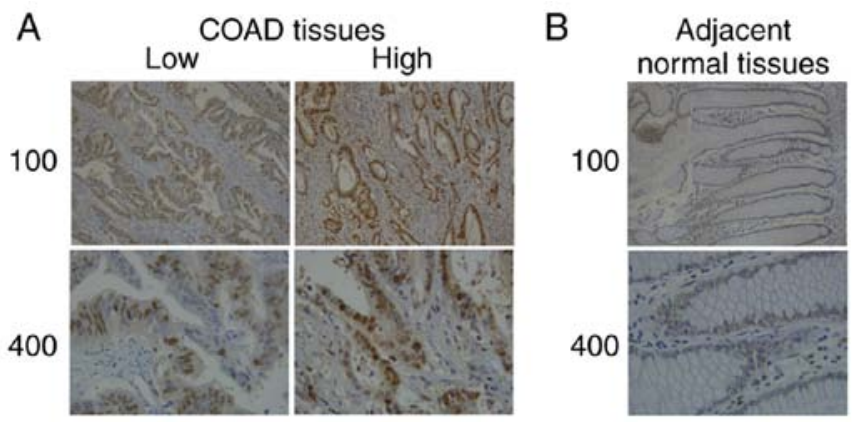

Figure 2. SMYD3 expression is upregulated in human COAD tissues (A) IHC assays were performed and the representative photographs of SMYD3 expression in human COAD tissues were presented (magnification, $\mathrm{x} 100$ and $\mathrm{x} 400$ ). (B) IHC assays were conducted and the results confirmed low COAD expression levels in the adjacent normal tissues (magnification, $\mathrm{x} 100$ and $\mathrm{x} 200$ ). SMYD3, SET and MYND domain-containing protein 3; COAD, colon adenocarcinoma.

expression. A total of 25 patients exhibited low SMYD3 expression and 67 patients exhibited high SMYD3 expression. Clinical features, including patient age, sex, tumor stage, tumor grade, lymph node metastasis and metastasis, of COAD patients were analyzed. The results demonstrated no significant correlation between SMYD3 expression and patient age $(\mathrm{P}=0.194)$, sex $(\mathrm{P}=0.846)$, tumor grade $(\mathrm{P}=0.267)$, lymph node metastasis $(\mathrm{P}=0.119)$ or metastasis $(\mathrm{P}=0.528)$ in patients with COAD. However, the data demonstrated that SMYD3 expression was correlated with tumor stage $(\mathrm{P}=0.038)$ in patients with COAD.

SMYD3 affects the proliferation and apoptosis of COAD cells in vitro. Since the results reported a potential association between SMYD3 expression and COAD, the effects of SMYD3 on COAD cells were examined. shRNA plasmids targeting SMYD3 were used to deplete the expression of SMYD3 in two types of colorectal cancer cells, HCT116 and HT-29. qPCR and immunoblotting assays were performed to detect SMYD3 expression in controls or SMYD3 shRNA-transfected HCT116 and HT-29 cells. According to the results, the mRNA and protein levels of SMYD3 were significantly decreased following the transfection shRNA plasmids in HCT116 and HT-29 cells compared with controls (Fig. 3A and B).

Subsequently, a series of in vitro assays were performed to evaluate the effects of SMYD3 on the progression of COAD. Colony formation assays demonstrated that SMYD3 ablation led to a significant decrease in the colony numbers of HCT116 and HT-29 cells compared with controls (Fig. 4A). Additionally, SMYD3 depletion resulted in a significantly decreased OD value at $570 \mathrm{~nm}$ wavelength in HCT116 and HT-29 cells compared with controls, as reported by MTT assays (Fig. 4B). These data confirmed the effects of SMYD3 on COAD cell proliferation. Furthermore, FCM assays were performed to detect the effects of SMYD3 on cell apoptosis and the cell cycle. The results demonstrated that SMYD3 knockdown significantly stimulated apoptosis in HCT116 and HT-29 cells (Fig. 4C). FCM results further demonstrated that SMYD3 depletion resulted in the arrest of cell cycle in these cells (Fig. 4D). In summary, these results confirmed the effects
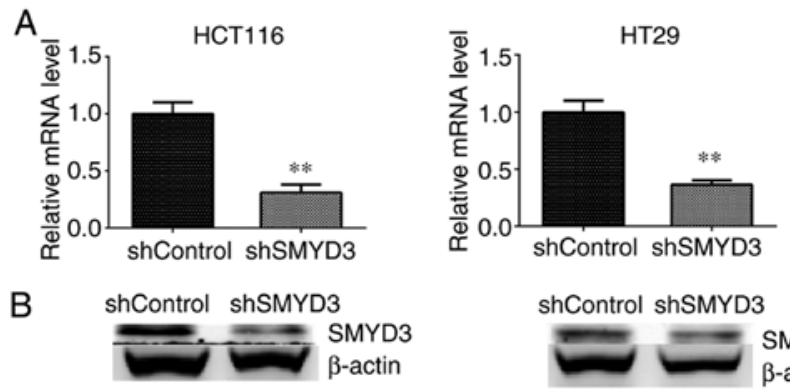

shControl shSMYD3
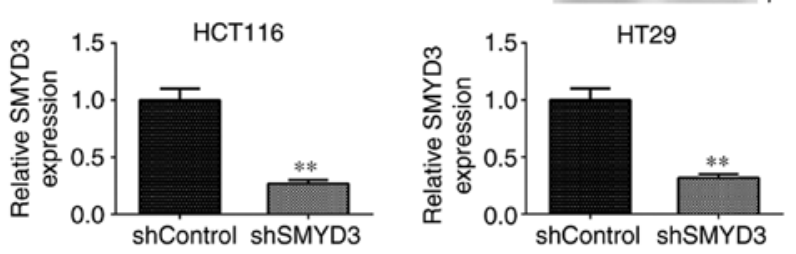

Figure 3. SMYD3 expression is decreased in shRNA-transfected HCT116 and HT-29 cells. (A) Quantitative PCR analysis revealed a significant decrease in SMYD3 mRNA post-transfection. (B) Immunoblotting demonstrated significant SMYD3 inhibition post-transfection. Results are presented as mean $\pm \mathrm{SD}$. ${ }^{* *} \mathrm{P}<0.01$. SMYD3, SET and MYND domain-containing protein 3 .

of SMYD3 on proliferation, apoptosis and the cell cycle in HCT116 and HT-29 cells in vitro.

SMYD3 promotes tumor growth of HCT116 and HT-29 cells in mice in vivo. To further confirm the previous in vitro data, whether SMYD3 contributed to tumor growth of COAD cells in an animal model was investigated. Briefly, HCT116 cells were infected with control or SMYD3 shRNA lentiviruses and were subcutaneously injected into nude mice. Tumors began forming after 14 days and tumor volumes were measured every 3 days. After 29 days, the mice were sacrificed and all tumors were isolated and photographed. The representative images of the tumors and the tumor growth curves are presented in Fig. 5A. Tumor volumes in the SMYD3-depleted groups were significantly decreased compared with controls (Fig. 5A). Furthermore, immunoblot assays were performed to detect SMYD3 expression in tumor tissues from mice in control and SMYD3-deplted depletion groups. The results demonstrated that SMYD3 expression levels were decreased in SMYD3-depleted tumor tissues compared with controls (Fig. 5B). Therefore, we hypothesized that SMYD3 promoted tumor growth of COAD cells in vivo.

\section{Discussion}

During early COAD, surgical resection is generally curative and patients with advanced COAD are often treated with surgery combined with chemoradiotherapy (23). Due to the high metastasis of cancer, advances in targeted therapy are required $(24,25)$. Targeted therapy is the most effective method to treat COAD (26). Numerous molecular targets of COAD have been discovered, including EGFR and sirtuin $6(27,28)$. Targeted therapy drugs, including Gefitinib and Erlotinib, are on the market or are currently in clinical trials (29). However, more therapeutic targets need to be developed to improve prognosis. The current study reported that a methyltransferase, 

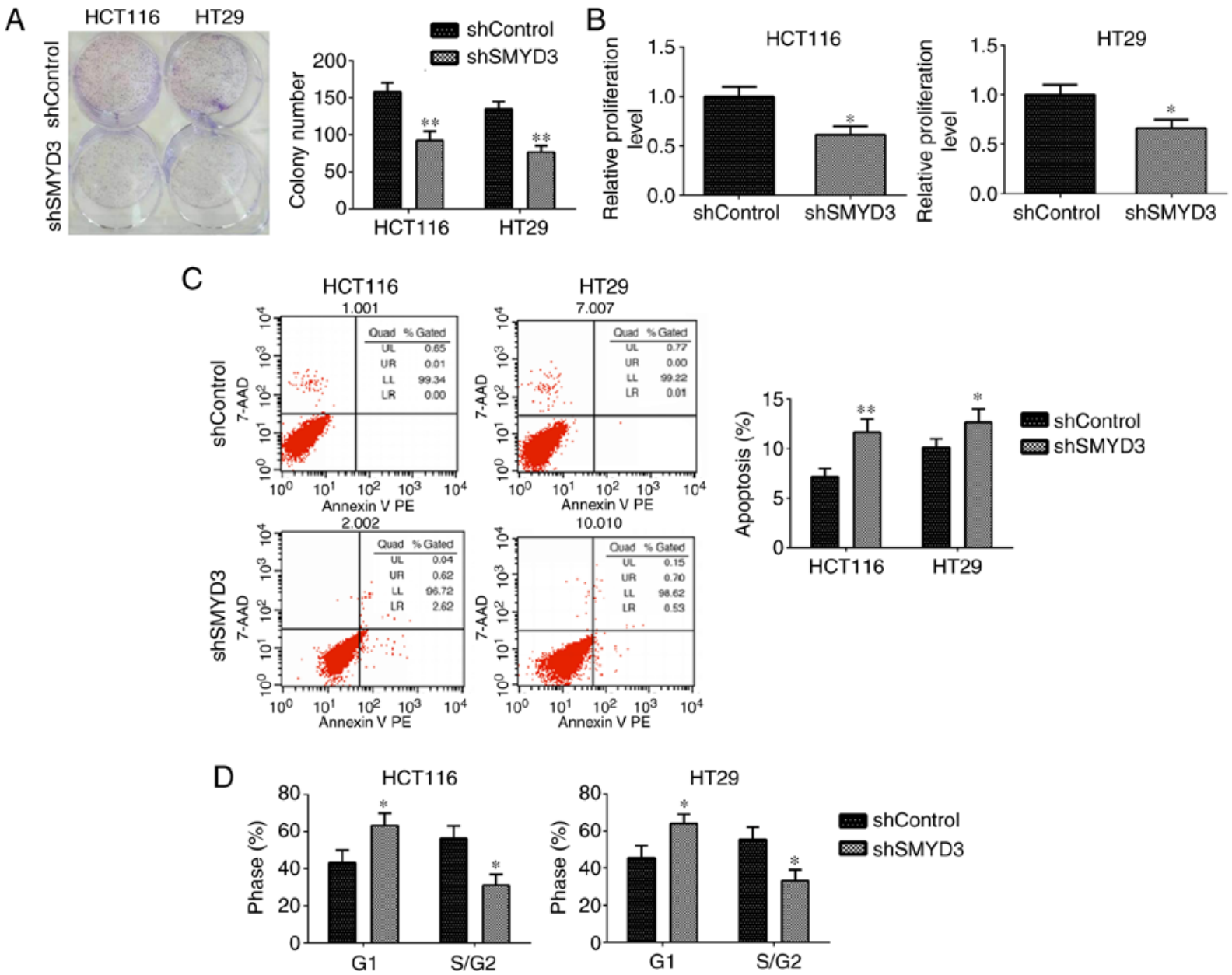

Figure 4. SMYD3 affects proliferation, apoptosis and the cell cycle in HCT116 and HT-29 cells in vitro. (A) Following transfection with control or SMYD3 shRNA plasmids, colony formation assays were performed and colony numbers were counted. (B) The results of MTT assays demonstrated decreased OD values at $570 \mathrm{~nm}$ wavelength post-transfection. (C) FCM assays were performed and the effects of SMYD3 on cell apoptosis were detected. (D) FCM assays were conducted and the effects of SMYD3 on the cell cycle were detected. Results are presented as mean \pm SD. ${ }^{*}<0.05$. ${ }^{* *} \mathrm{P}<0.01$. SMYD3, SET and MYND domain-containing protein 3 ; sh, short hairpin; FCM, flow cytometry.

A
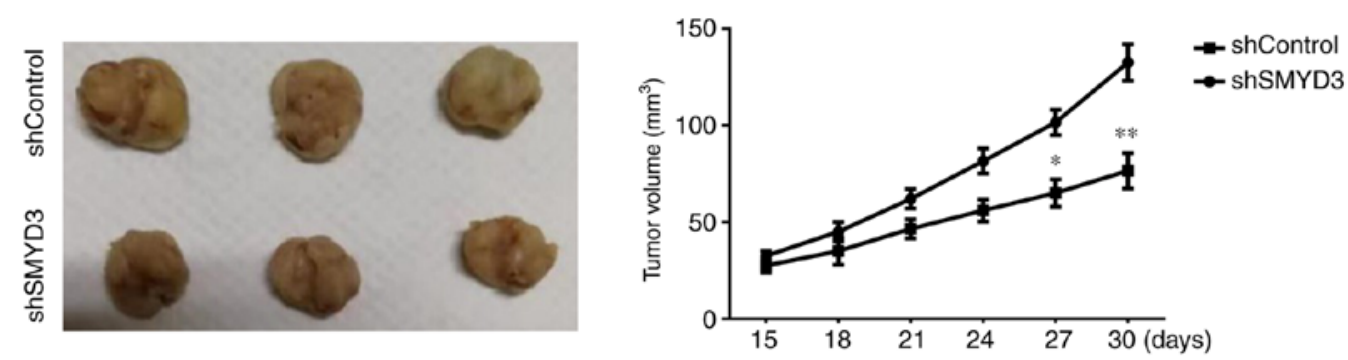

B

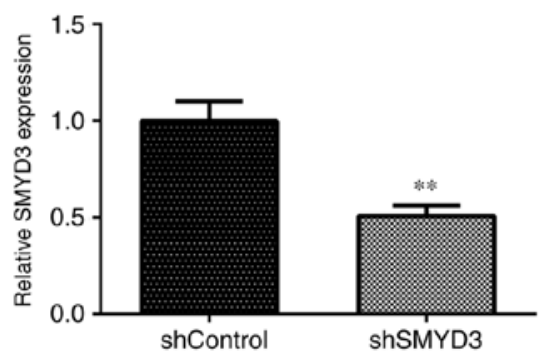

Figure 5. SMYD3 stimulates tumor growth of COAD cells in mice in vivo. (A) HCT116 cells transfected with control or SMYD3 shRNA lentiviruses were subcutaneously implanted into nude mice. Tumors began forming 14 days post-implantation and tumor volumes were measured every 3 days. (A) After 29 days, all tumors were excised and the growth curves were analyzed in the SMYD3-depleted and control groups. (B) Immunoblotting revealed the expression levels of SMYD3 in control and SMYD3-depleted tumors. Results are presented as mean \pm SD. Scale bar, $5 \mathrm{~mm}$. ${ }^{*} \mathrm{P}<0.05 .{ }^{* *} \mathrm{P} 0.01$. SMYD3, SET and MYND domain-containing protein 3; COAD, colon adenocarcinoma; sh, short hairpin. Scale bar indicates $5 \mathrm{~mm}$. 
SMYD3, was exhibited significantly high expression in human COAD tissues compared with controls. Furthermore, the results that confirmed SMYD3 was correlated with prognosis and clinical feature of patients with COAD. Therefore, we hypothesized that SMYD3 may serve as a novel and promising molecular target for COAD treatment.

Through the investigation of SMYD3 on COAD progression in vitro, colony formation, MTT and FCM assays were performed and the results demonstrated that the depletion of SMYD3 impaired proliferation, stimulated apoptosis and induced cell cycle arrest in COAD cells. Furthermore, in vivo assays reported that SMYD3 contributed to tumor growth in mice, which was consistent with the in vitro data. Therefore, the results confirmed that SMYD3 regulated COAD progression by mediating cell proliferation, apoptosis and the cell cycle. Similarly, several previous studies have demonstrated the effects of SMYD3 on cancer progression (30-33). SMYD3 stimulated epithelial ovarian cancer metastasis by suppressing p53 protein stability and promoting p53 ubiquitination (30). Additionally, SMYD3 was associated with the proliferation and apoptosis of ovarian cancer by regulating methylation (18). SMYD3 inhibition also suppressed cell proliferation, migration and invasion of pancreatic cancer cells (34). These previous studies, together with the results of the current study, indicated the critical role of SMYD3 in tumor growth and development. Therefore, developing novel inhibitors for SMYD3 may be an effective target for cancer treatment.

Post-translational modifications mediated various cellular processes, including mitosis and cell migration, by regulating protein function $(13,35)$. As a methyltransferase, SMYD3 methylated H3K4 and H4K20, altering the expression of downstream genes (36). Additionally, SMYD3 methylated non-histone proteins, regulating body development and tumor progression (37). Furthermore, SMYD3 promoted myogenesis by activating the myogenin regulatory network (38). The current study demonstrated that SMYD3 affected proliferation and apoptosis of COAD cells in vitro and tumor growth in mice in vivo. We hypothesized that SMYD3 likely influenced the development of COAD by regulating the methylation of key tumor-regulated proteins. However, further experiments are required to confirm this hypothesis. Additionally, whether SMYD3 affects the migration and invasion of COAD cells deserves further investigation.

The SMYD methyltransferase family is known to affect the progression of several types of tumors, such as breast cancer and lung cancer (39). SMYD1 has been reported to be associated with several solid tumors, including breast cancer (40). Additionally, SMYD2 exhibited high expression in multiple types of tumors, including gastric cancer and colorectal cancer, and affected the migration and invasion of tumor cells $(41,42)$. Combined with the results of the current study, we hypothesized that the development of inhibitors targeting SMYD family proteins has potential advantages for cancer targeted therapy.

In summary, the results of the current study demonstrated that SMYD3 was highly expressed in human COAD tissues and was correlated with the prognosis and a clinical feature, tumor stage, in patients with COAD. SMYD3 affected cell growth by mediating cell proliferation, apoptosis and the cell cycle in vitro, and tumor growth in mice in vivo. Therefore, we hypothesize that SMYD3 has potential to serve as a promising COAD therapeutic target.

\section{Acknowledgements}

Not applicable.

\section{Funding}

No funding was received.

\section{Availability of data and materials}

The datasets used and/or analyzed during the current study are available from the corresponding author on reasonable request.

\section{Authors' contributions}

FRY, ZBW and RZY performed the molecular biology experiments and drafted the manuscript. QHG and BL participated in the design of the study and performed statistical analysis. FRY, JHZ and ZL conceived the current study, participated in design and coordination, and assisted in drafting the manuscript. All authors read and approved the final manuscript.

\section{Ethics approval and consent to participate}

All procedures performed in the current study were approved by the Ethics Committee of Tianjin Baodi Hospital, Tianjin, China. Written informed consent was obtained from all patients or their families.

\section{Patient consent for publication}

Not applicable.

\section{Competing interests}

The authors declare that they have no competing interests.

\section{References}

1. Chang HK, Gen Y, Yeo SG, Jung JH and Park DC: Primary adenocarcinoma with choriocarcinomatous differentiation of the sigmoid colon misdiagnosed choriocarcinoma of the uterus: Case report and review of the literature. Eur J Gynaecol Oncol 40: 468-470, 2019.

2. Lian J, Xia L, Chen Y, Zheng J, Ma K, Luo L and Ye F: Aldolase $\mathrm{B}$ impairs DNA mismatch repair and induces apoptosis in colon adenocarcinoma. Pathol Res Pract 215: 152597, 2019.

3. Kai K, Hidaka H, Nakamura T, Ueda Y, Marutsuka K, Ikeda T and Nanashima A: A case of poorly differentiated adenocarcinoma with lymphoid stroma originated in the ascending colon diagnosed as lymphoepithelioma-like carcinoma. Clin J Gastroenterol 13: 538-544, 2020.

4. Li Z, Tan H, Yu H, Deng Z, Zhou X and Wang M: DNA methylation and gene expression profiles characterize epigenetic regulation of lncRNAs in colon adenocarcinoma. J Cell Biochem 121: 2406-2415, 2020.

5. Bottino C, Peserico A, Simone C and Caretti G: SMYD3: An oncogenic driver targeting epigenetic regulation and signaling pathways. Cancers (Basel) 12: 142, 2020.

6. Dong S and Zhang P: Advances of histone methyltransferase SMYD3 in tumors. Zhongguo Fei Ai Za Zhi 17: 689-694, 2014 (In Chinese).

7. Eberle CA, Zayas M, Stukalov A, Pichlmair A, Alvisi G, Muller AC, Bennett KL, Bartenschlager R and Superti-Furga G: The lysine methyltransferase SMYD3 interacts with hepatitis $C$ virus NS5A and is a negative regulator of viral particle production. Virology 462-463: 34-41, 2014. 
8. Chen YJ, Tsai CH, Wang PY and Teng SC: SMYD3 promotes homologous recombination via regulation of H3K4-mediated gene expression. Sci Rep 7: 3842, 2017.

9. Wang Q, Jiang Y, Luo X, Wang C, Wang N, He H, Zhang T and Chen L: Chitooligosaccharides modulate glucose-lipid metabolism by suppressing SMYD3 pathways and regulating gut microflora. Mar Drugs 18: 69, 2020.

10. Chen J, He Z, Yuan Y, Huang F, Luo B, Zhang J, Pan T, Zhang H and Zhang J: Host factor SMYD3 is recruited by Ebola virus nucleoprotein to facilitate viral mRNA transcription. Emerg Microbes Infect 8: 1347-1360, 2019.

11. Al-Eitan LN and Rababa'h DM: Correlation between a variable number tandem repeat (VNTR) polymorphism in SMYD3 gene and breast cancer: A genotype-phenotype study. Gene 728: 144281, 2020.

12. Chen D, Liu L, Luo X, Mu A, Yan L, Chen X, Wang L, Wang N, $\mathrm{He} \mathrm{H}$, Zhou H and Zhang T: Effect of SMYD3 on the microRNA expression profile of MCF-7 breast cancer cells. Oncol Lett 14: 1831-1840, 2017.

13. Cock-Rada AM, Medjkane S, Janski N, Yousfi N, Perichon M, Chaussepied M, Chluba J, Langsley G and Weitzman JB: SMYD3 promotes cancer invasion by epigenetic upregulation of the metalloproteinase MMP-9. Cancer Res 72: 810-820, 2012.

14. Colon-Bolea $\mathrm{P}$ and Crespo P: Lysine methylation in cancer: SMYD3-MAP3K2 teaches us new lessons in the Ras-ERK pathway. Bioessays 36: 1162-1169, 2014.

15. Dong QQ, Wang QT, Wang L, Jiang YX, Liu ML, Hu HJ, Liu Y, Zhou H, He HP, Zhang TC and Luo XG: SMYD3-associated pathway is involved in the anti-tumor effects of sulforaphane on gastric carcinoma cells. Food Sci Biotechnol 27: 1165-1173, 2018

16. Fei X, Ma Y, Liu X and Meng Z: Overexpression of SMYD3 is predictive of unfavorable prognosis in hepatocellular carcinoma. Tohoku J Exp Med 243: 219-226, 2017.

17. Fenizia C, Bottino C, Corbetta S, Fittipaldi R, Floris P, Gaudenzi G, Carra S, Cotelli F, Vitale G and Caretti G: SMYD3 promotes the epithelial-mesenchymal transition in breast cancer. Nucleic Acids Res 47: 1278-1293, 2019.

18. Jiang Y, Lyu T, Che X, Jia N, Li Q and Feng W: Overexpression of SMYD3 in ovarian cancer is associated with ovarian cancer proliferation and apoptosis via methylating H3K4 and H4K20. J Cancer 10: 4072-4084, 2019.

19. Li B, Pan R, Zhou C, Dai J, Mao Y, Chen M, Huang T, Ying X, Hu H, Zhao J, et al: SMYD3 promoter hypomethylation is associated with the risk of colorectal cancer. Future Oncol 14: 1825-1834, 2018.

20. Liu C, Wang C, Wang K, Liu L, Shen Q, Yan K, Sun X, Chen J, Liu J, Ren H, et al: SMYD3 as an oncogenic driver in prostate cancer by stimulation of androgen receptor transcription. J Natl Cancer Inst 105: 1719-1728, 2013.

21. Wang Y, Xie BH, Lin WH, Huang YH, Ni JY, Hu J, Cui W, Zhou J, Shen L, Xu LF, et al: Amplification of SMYD3 promotes tumorigenicity and intrahepatic metastasis of hepatocellular carcinoma via upregulation of CDK2 and MMP2. Oncogene 38: 4948-4961, 2019.

22. Livak KJ and Schmittgen TD: Analysis of relative gene expression data using real-time quantitative PCR and the 2(-Delta Delta C(T)) method. Methods 25: 402-408, 2001.

23. Nowak A, Zaklos-Szyda M, Zyzelewicz D, Koszucka A and Motyl I: Acrylamide decreases cell viability, and provides oxidative stress, DNA damage, and apoptosis in human colon adenocarcinoma cell line caco-2. Molecules 25: 368, 2020.

24. Yang Y, Zhao Z, Xie C and Zhao Y: Dual-targeting liposome modified by glutamic hexapeptide and folic acid for bone metastatic breast cancer. Chem Phys Lipids 228: 104882, 2020.

25. Zhao Z, Zhao Y, Xie C, Chen C, Lin D, Wang S, Lin D, Cui X, Guo $\mathrm{Z}$ and Zhou J: Dual-active targeting liposomes drug delivery system for bone metastatic breast cancer: Synthesis and biological evaluation. Chem Phys Lipids 223: 104785, 2019.

26. Ruan GT, Zhu LC, Gong YZ, Liao XW, Wang XK, Liao C, Wang S, Yan L, Xie HL, Zhou X, et al: The diagnosis and prognosis values of WNT mRNA expression in colon adenocarcinoma. J Cell Biochem 121: 3145-3161, 2020.
27. Ni X, Ding Y, Yuan H, Shao J, Yan Y, Guo R, Luan W and Xu M: Long non-coding RNA ZEB1-AS1 promotes colon adenocarcinoma malignant progression via miR-455-3p/PAK2 axis. Cell Prolif 53: e12723, 2020

28. Chang CL, Yuan KS, Wu ATH and Wu SY: Adjuvant therapy for high-risk stage II or III colon adenocarcinoma: A propensity score-matched, nationwide, population-based cohort study. Cancers (Basel) 11: 2003, 2019.

29. Chan KWK, Chung HY and Ho WS: Anti-tumor activity of atractylenolide I in human colon adenocarcinoma in vitro. Molecules 25: 212, 2020.

30. Zhang L, Jin Y, Yang H, Li Y, Wang C, Shi Y and Wang Y: SMYD3 promotes epithelial ovarian cancer metastasis by downregulating $\mathrm{p} 53$ protein stability and promoting $\mathrm{p} 53$ ubiquitination. Carcinogenesis 40: 1492-1503, 2019.

31. Liu N, Sun S and Yang X: Prognostic significance of stromal SMYD3 expression in colorectal cancer of TNM stage I-III. Int J Clin Exp Pathol 10: 8901-8907, 2017.

32. Liu TT, Xu H, Gao WP, Zhang SX, Zhou XH, Tang J and Liu QN: SET and MYND domain-containing protein 3 (SMYD3) polymorphism as a risk factor for susceptibility and poor prognosis in ovarian cancer. Med Sci Monit 22: 5131-5140, 2016.

33. Lyu T, Jiang Y, Jia N, Che X, Li Q, Yu Y, Hua K, Bast RC Jr and Feng W: SMYD3 promotes implant metastasis of ovarian cancer via H3K4 trimethylation of integrin promoters. Int J Cancer 146: 1553-1567, 2020.

34. Zhu CL and Huang Q: Overexpression of the SMYD3 promotes proliferation, migration, and invasion of pancreatic cancer. Dig Dis Sci 65: 489-499, 2020.

35. Bai H, Li Y, Gao H, Dong Y, Han P and Yu H: Histone methyltransferase SMYD3 regulates the expression of transcriptional factors during bovine oocyte maturation and early embryonic development. Cytotechnology 68: 849-859, 2016.

36. Sun J, Shi F and Yang N: Exploration of the substrate preference of lysine methyltransferase SMYD3 by molecular dynamics simulations. ACS Omega 4: 19573-19581, 2019.

37. Van Aller GS, Reynoird N, Barbash O, Huddleston M, Liu S, Zmoos AF, McDevitt P, Sinnamon R, Le B, Mas G, et al: Smyd3 regulates cancer cell phenotypes and catalyzes histone $\mathrm{H} 4$ lysine 5 methylation. Epigenetics 7: 340-343, 2012.

38. Codato R, Perichon M, Divol A, Fung E, Sotiropoulos A, Bigot A, Weitzman JB and Medjkane S: The SMYD3 methyltransferase promotes myogenesis by activating the myogenin regulatory network. Sci Rep 9: 17298, 2019.

39. Song J, Liu Y, Chen Q, Yang J, Jiang Z, Zhang H, Liu Z and Jin B: Expression patterns and the prognostic value of the SMYD family members in human breast carcinoma using integrative bioinformatics analysis. Oncol Lett 17: 3851-3861, 2019.

40. Tan X, Rotllant J, Li H, De Deyne P and Du SJ: SmyD1, a histone methyltransferase, is required for myofibril organization and muscle contraction in zebrafish embryos. Proc Natl Acad Sci USA 103: 2713-2718, 2006.

41. Yan L, Ding B, Liu H, Zhang Y, Zeng J, Hu J, Yao W, Yu G, An R, Chen Z, et al: Inhibition of SMYD2 suppresses tumor progression by down-regulating microRNA-125b and attenuates multi-drug resistance in renal cell carcinoma. Theranostics 9: 8377-8391, 2019.

42. Ohtomo-Oda R, Komatsu S, Mori T, Sekine S, Hirajima S, Yoshimoto S, Kanai Y, Otsuji E, Ikeda E and Tsuda H: SMYD2 overexpression is associated with tumor cell proliferation and a worse outcome in human papillomavirus-unrelated nonmultiple head and neck carcinomas. Hum Pathol 49: 145-155, 2016.

This work is licensed under a Creative Commons Attribution-NonCommercial-NoDerivatives 4.0 International (CC BY-NC-ND 4.0) License. 